\title{
Evaluation of Digital Filters for Rejecting Discrete Spectral Interference in On-site PD Measurements
}

\author{
V. Nagesh and B. I. Gururaj \\ Dept. of HV Engg., Indian Institute of Science, \\ Bangalore, India
}

\begin{abstract}
While Partial Discharge (PD) measurements are widely used in testing power apparatus after manufacture, there is now a trend to extend them to on-site measurements. The major problem encountered in the latter measurements is the strong coupling of external noises particularly from discrete spectral interferences (DSI) e.g. broadcasting stations as well as impulsive disturbances. A critical study of the performance of several digital filters for rejecting DSI is reported. The filters are evaluated with particular reference to distortion introduced on successive PD pulses. A new digital filtering method based on a cascaded 2nd order IIR lattice notch filter has been proposed. The study showed that, for similar performance, methods based on linear prediction required longer filter orders when compared to cascaded IIR notch filters. The nonadaptive methods were found to be stable against impulsive disturbances while adaptive methods were unstable. The new method proposed in this paper gave the best performance, low distortion to a sequence of PD pulses, good stability and fast filtering time. The performance at higher sampling rates, even with PD pulses of long widths was also found to be superior. Further, the method is implementable using fixed-point arithmetic leading to faster processing. Hence, the proposed method can be suggested for on-site PD measurements.
\end{abstract}

\section{INTRODUCTION}

$\mathrm{P}$ ARTIAL discharge (PD) measurement is a very powerful tool to detect defects in the insulation structure during manufacture and to indicate design inadequacies. While these measurements are now carried out in shielded laboratories with filtered power supplies, there is a trend to extend these to energized equipment such as transformers. The major problem with on-site measurement is the strong coupling of various noises into the PD measurement circuit. These noises greatly reduce the sensi- tivity of the measured PD and even prevent meaningful measurements. Although bandwidths below $500 \mathrm{kHz}$ are chosen in the present-day PD detectors to reduce external noise, their use in on-site conditions cannot reject all the noises present. Also, better diagnostics can be achieved by increasing the PD pulse resolution, i.e. by having a larger bandwidth.

In order to increase the bandwidth and simultaneously reduce the noise effects, flexible noise reduction methods have to be used. These are very difficult to be implemented using analog circuits. Fortunately, with the 
advent of VLSI, fast and accurate A/D converters have become commercially available and the PD signal can be digitized. The digital data can be processed using digital signal processing techniques for noise reduction.

Common interference that is coupled into on-site measuring circuits in the frequency range $\leqslant 1.5 \mathrm{MHz}$ is caused by

1. Discrete spectral interference (DSI), e.g. communication and AM Radio emissions.

2. Repetitive pulses, e.g. from power electronics or other periodic switching.

3. Random pulses, e.g. from infrequent switching operations or lightning.

4. Other noise sources such as ambient and amplifier noise.

In addition, corona discharges on external metal parts of other substation apparatus as well as internal PD can cause noise. However, this can be minimized by proper choice of coupling circuits and grounding arrangement.

The subject of digital filters is very mature and is being applied in fields such as communication technology, speech enhancement, radar, etc. and a variety of structures and algorithms are available to design and realize the filters. Only two of these techniques have been utilized in on-site PD measurements. In 1988, Feser et al. [1] have suggested a FFT-based approach to eliminate DSI; however, it is computationally intensive. As an alternative, Borsi and Hartje [2] in 1989, used an adaptive digital filter to suppress the DSI. A critical study and evaluation of all the filtering methods, which are efficient in identifying and rejecting DSI in PD measurements is reported in this paper. The performance of suitable digital filters has been compared including the techniques proposed in [1] and [2]. In addition, a new and efficient method for rejecting DSI has been identified and proposed in this work. The methods for rejecting pulse interference are being reported separately.

\section{DESIGN OF DIGITAL FILTERS}

$\mathrm{T}$ HE spectral characteristics of the DSI and PD sig1 nals are very different; the former has a narrow-band spectrum centered around dominant frequencies, while the latter has a broad spectrum. This difference is used in designing digital filters for rejecting the DSI.
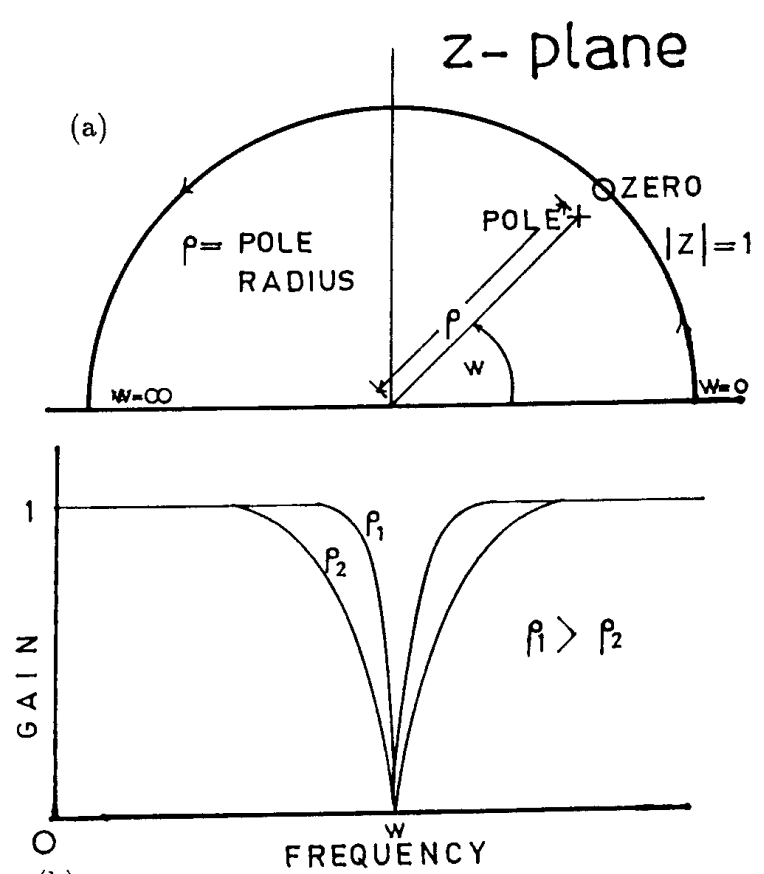

(b)

Figure 1.

(a) Pole-zero plot of 2nd order IIR notch filter.

(b) Frequency response of 2 nd order IIR notch filter.

\subsection{AVAILABLE DIGITAL FILTERS FOR REJECTING DSI}

In a broad sense, the methods for realization of digital filters can be divided into two classes: nonrecursive and recursive. The nonrecursive filters are also called finite impulse response (FIR) filters. The FIR filters considered in this work are transversal, multistage lattice and linearphase filters. A detailed description of these FIR filters is given by Oppenheim and Schafer [3]. The recursive filters are called as infinite impulse response (IIR) filters [3]. A particularly efficient IIR filter for rejecting DSI is the IIR notch filter. In this filter, the zeros are made to lie on the unit circle (complex $z$-plane) at an angle equal to the input discrete frequency which has to be rejected. The pole is constrained to lie inside the unit circle, at the same angle, and as close as possible to the zero. A zero pole of such a 2nd order IIR notch filter is shown in Figure 1(a). As the pole radius approaches unity, the bandwidth of the notch reduces as shown in Figure 1(b), due to the narrow bandwidths achievable, they are very efficient in rejecting DSI. 


\subsection{METHODS OF OBTAINING FILTER COEFFICIENTS}

If the frequency of interest is limited to $1 \mathrm{MHz}$, then the sampling period has to be $\leqslant 500 \mathrm{~ns}$. At this sampling rate, real-time processing is difficult due to hardware limitations. Hence, block processing methods have to be adopted. Thus, a long record containing the PD signal (extending over a few power frequency cycles) is digitized and later processed to remove the interferences. Since the time frame is small and the DSI bandwidth is narrow, the record can be considered as stationary. Therefore methods applicable for stationary signals can be used in this situation for obtaining the filter coefficients.

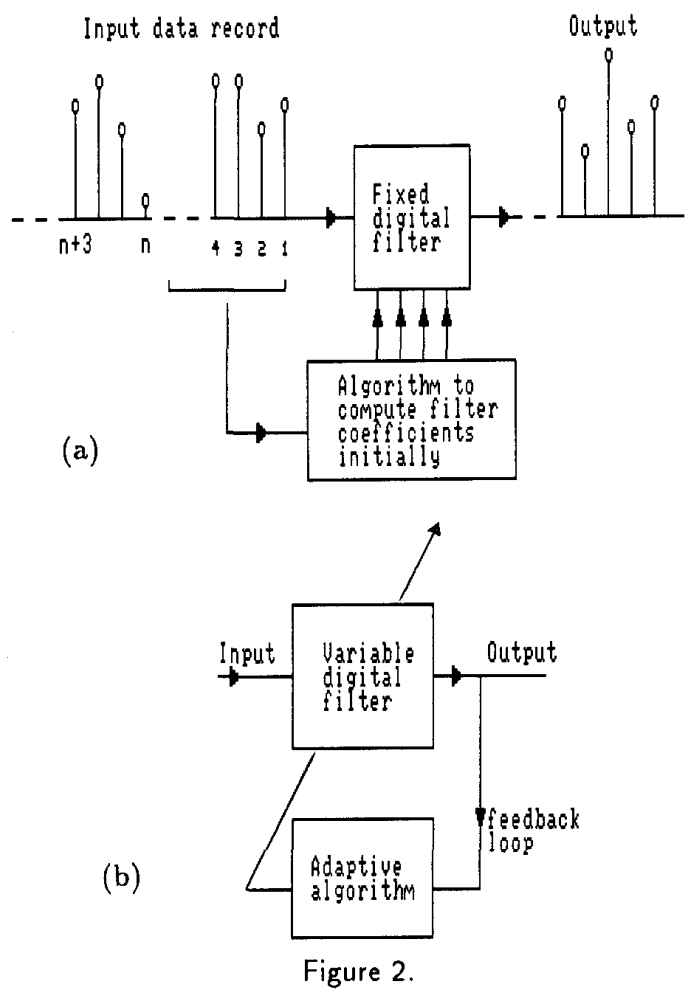

Filtering methods. (a) Nonadaptive. (b) Adaptive

The coefficients of the required digital filter can be obtained by different methods. These can be divided as nonadaptive and adaptive. In nonadaptive methods, Figure 2(a), a small block of the record is initially used and its characteristics are calculated. Then, a fixed digital filter is designed for eliminating the DSI optimally. In contrast, the adaptive methods have a closed loop configuration (Figure 2(b)) in which the filter coefficients are varied to reduce the DSI. Here, the pertinent interference characteristics are not estimated explicitly; rather, the
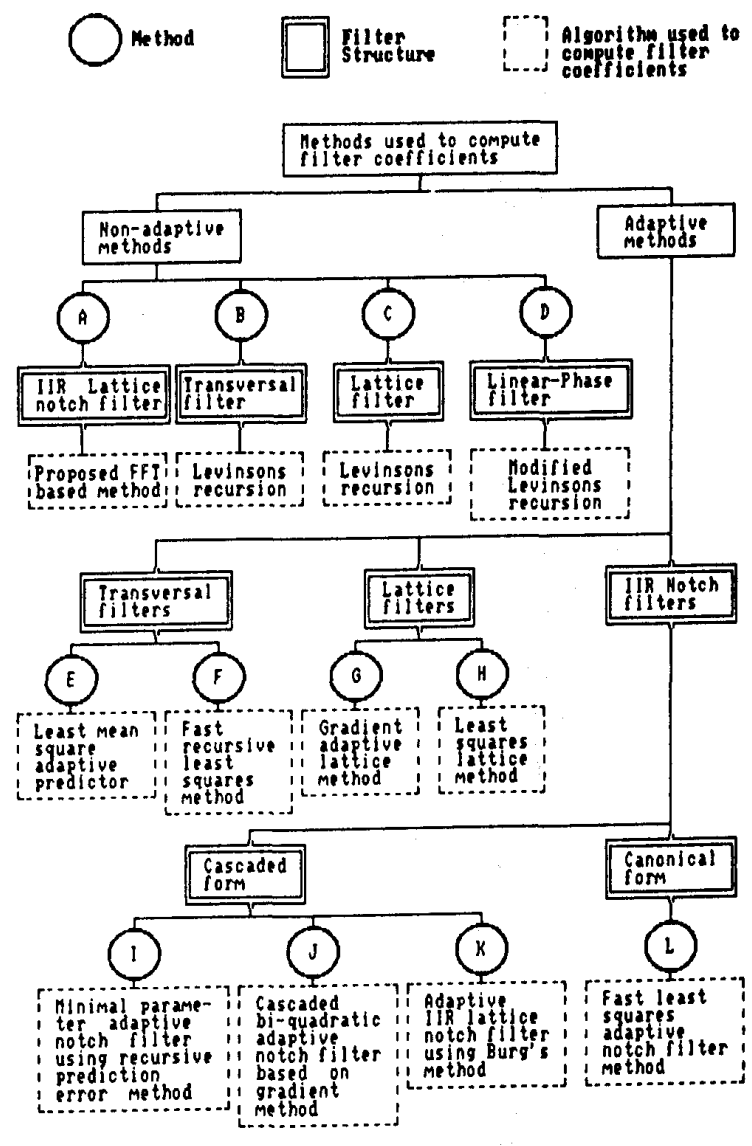

Figure 3.

Classification of different digital filtering methods for rejection of DSI

filter accomplishes the objective by automatically updating the filter coefficients with the availability of each new sample of the data. After processing a number of samples in this way, the algorithm evolves the optimum filter coefficients after which the adaptation can be stopped, if necessary. A detailed study of the applicable filters for eliminating DSI was made. The resulting classification is shown in Figure 3. Only the essential details of these methods are given in this paper in the interest of brevity.

\section{DIGITAL SIMULATION SETUP \\ 3.1 NEED FOR SYNTHESIS OF INPUT SIGNAL}

Tor studying the performance of any digital filter for this application, it is necessary to know both the input 
and output signal sequences. Hence, the input signal to the digital filter containing the PD pulses and DSI needs to be synthesized in a realistic manner. This approach is widely used in signal processing work. Another advantage of this approach is that it facilitates reproducibility of the input signal by other researchers. Furthermore, the number of DSI and its amplitude at any location where on-site PD measurements are performed, depend on the service areas of the emitters; these also vary with respect to time: day or night. Feser et al. [1] and Borsi et al. [2] have reported the presence of several emitters. Hence, in order to evaluate the different filtering methods when many emitters are present, the DSI signals were synthetically generated. In addition, the ever present background noise (which exists even in shielded laboratories) has to be modeled, because many digital filters behave strangely in their presence. In the absence of definitive statistics, this noise is modeled as white noise.

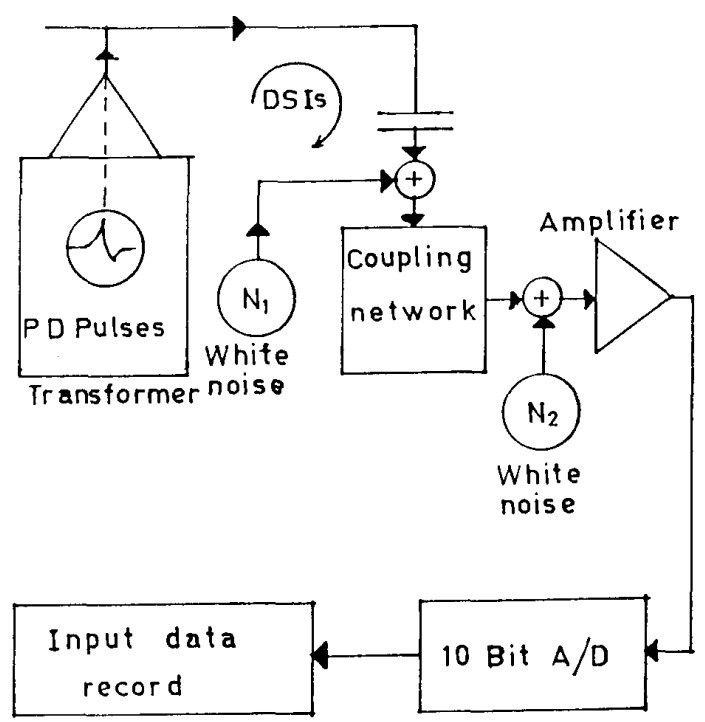

Figure 4. Schematic of on-site PD test setup.

\subsection{CHOICE OF SIMULATION PARAMETERS}

The block diagram of an on-site PD measurement system is shown in Figure 4 and the same is simulated. The input signal is synthesized by superposing pulses of known shape with other interferences like DSI, random impulsive disturbances and a small background noise. The parameters chosen for simulation are discussed below.

\subsubsection{BACKGROUND NOISE}

The white noise generators $\mathrm{N}_{1}$ and $\mathrm{N}_{2}$ are used to simulate the background noise only. It is uncorrelated with the
DSI and remains even after filtering the DSI. The white noise generator $\mathrm{N}_{1}$ simulates external ambient noise (other than the DSI) and $\mathrm{N}_{2}$ simulates the small noise of the amplifier. The ratio of the power of noise $N_{1}$ to the power of noise $\mathrm{N}_{2}$ at the input to the digital filter was 1.3.

\subsubsection{PD PULSES}

The number, shapes and time intervals of PD signals measured at terminals of $\mathrm{HV}$ apparatus vary widely according to the type of $\mathrm{PD}$, coupling circuit and transmission path. A good filter should reproduce these PD pulses with minimum distortion even when the height, duration and time intervals between the pulses are small. In order to consider realistic PD pulses in a capacitive apparatus, an exponentially decaying pulse with time constant of $50 \mathrm{~ns}$ (similar to that in oil-paper insulation) has been used. Other PD pulse shapes such as exponentially decay with time constant $\leqslant 2 \mu$ s (oil gaps), as well as oscillatory pulses (transformers and cable systems) have been considered in Section 5.2. To simulate the effect of the coupling circuit on this pulse it was convolved with the impulse response of 1st order Butterworth bandpass filter ( 30 to $300 \mathrm{kHz}$ ). The method used is similar to that suggested by Zaengl et al. [17], except that they have used a 2nd order bandpass filter. The use of 2nd or higher order bandpass filter would have resulted only in slightly longer output pulse widths and rise times.

In order to study the pulse resolution performance of the filter, a sequence of pulses has to be used. The time interval between successive PD pulses in a practical setup is random. Following Steiner and Weeks [18], who modeled it as a Poisson shot noise process, the time interval between PD pulses are generated here by using a Poisson distribution with a mean of $10 \mu \mathrm{s}$. The amplitude of all the PD pulses were chosen to be equal. The number of pulses were chosen to be 40 in each half cycle to represent considerable PD activity. A PD pulse height of approximately three times the white noise $\left(N_{1}\right.$ and $\left.N_{2}\right)$ level (without the DSI) was chosen to represent an acceptable sensitivity as in a shielded laboratory.

Table 1.

Power levels of DSI used in simulation

\begin{tabular}{|l|cccccccc|}
\hline \hline$f, \mathrm{kHz}$ & 200 & 300 & 450 & 500 & 620 & 700 & 800 & 980 \\
$\mathrm{INR}, \mathrm{dB}$ & 1.77 & 10.0 & -0.75 & 2.94 & 10.4 & -6.2 & 2.1 & -6.2 \\
\hline \hline
\end{tabular}

3.2.3 DSI

To understand first the effect of DSI, the input signal is synthesized with only modulated DSI, PD pulse and back- 
ground noise. The DSI generator synthesized eight AM sine waves with $40 \%$ modulation with a constant modulating frequency of $1 \mathrm{kHz}$. The ratio of the power of any DSI to the background noise $\left(\mathrm{N}_{1}\right.$ and $\left.\mathrm{N}_{2}\right)$ power at the input to the digital filter is named the interference to noise ratio (INR). The INR for the eight different DSI used in the simulation are given in Table 1 . The indicated power levels were chosen to represent a worst-case scenario. Hence, in addition to a few DSI with high INR near the center of the frequency spectrum, many DSI with low INR, well distributed in the frequency spectrum were added. It is very difficult to detect and reject DSI with low INR and more so if the frequencies are very near the Nyquist frequency.

\subsubsection{IMPULSIVE DISTURBANCES}

In on-site measurements, it is difficult to avoid the presence of large random impulsive disturbances. This has been reported recently by Konig et al. [16]. Measurements done by the authors using using wide-band antenna also have confirmed this. Therefore, the effect of impulsive disturbances should be considered in any simulation. Details in this regard are given in Section 4.3.

\subsection{BASIS FOR COMPARISON OF FILTER PERFORMANCE}

As mentioned earlier, a good filter should reject as much as possible the DSI, without distorting the PD pulse heights, shapes and the time intervals between the pulses. In order to compare the performance of digital filters identified here, it is necessary to have a measure for the distortion produced in filtering. The distortion in the filtered output is that of the PD pulse introduced by the digital filter and the residual DSI present. The normalized mean square error (MSE) between the input and output sequences $X$ and $Y$ is defined as

$$
\text { MSE }=\frac{\sum_{i=0}^{n}[Y(i)-X(i)]^{2}}{n \sigma_{n}^{2}}
$$

where $n$ is the number of samples for which $X$ is defined (PD pulse region), and $\sigma_{n}^{2}$ the total power of background noise $\left(\mathrm{N}_{1}\right.$ and $\left.\mathrm{N}_{2}\right)$ at the input to the filter was chosen as the normalization factor. If the filter is ideal in rejecting all the DSI, then the MSE will approach the power of noise at the filter input i.e., tend to unity. The MSE will increase if residual DSI are present or if the PD pulses at the output are distorted. MSE measure was chosen in this study because it is very sensitive to the presence of residual DSI and PD pulse distortion; in addition, its calculation is easy. As the MSE was found to vary slightly when different input sequences (generated with different random number sets) are processed by the digital filters, the mean MSE of five such input signals were taken as representative values.

Table 2.

Procedure and parameters used for simulating all the filters

\begin{tabular}{|c|c|c|}
\hline Method & Ref. & Procedure and parameters \\
\hline$\overline{\mathrm{A}}$ & Fig. 5 & $\begin{array}{l}\text { The DSI frequencies were estimated } \\
\text { using [6], the coeff. } K_{0} \text { is calculated for } \\
\text { each stage of the cascaded lattice IIR } \\
\text { notch filter } \alpha=0.98\end{array}$ \\
\hline B & {$[7]$} & $\begin{array}{l}\text { The auto-correlation lags were comput- } \\
\text { ed using the first } 4000 \text { samples }\end{array}$ \\
\hline $\mathrm{C}$ & [7] & $\begin{array}{l}\text { The auto-correlation lags were comput- } \\
\text { ed using the first } 4000 \text { samples }\end{array}$ \\
\hline $\mathrm{D}$ & {$[8]$} & $\begin{array}{l}\text { The auto-correlation lags were comput- } \\
\text { ed using the first } 4000 \text { samples }\end{array}$ \\
\hline $\mathrm{E}$ & [7] & $\mu=0.002$ \\
\hline $\mathrm{F}$ & [9] & $\begin{array}{l}\text { Double precision arithmetic was used } \\
\text { for stability, } \lambda=1.0\end{array}$ \\
\hline G & {$[10]$} & $\begin{array}{l}\lambda=0.9995, \beta=1, K_{f}=K_{b} \text { for all } \\
\text { stages }\end{array}$ \\
\hline $\mathrm{H}$ & {$[10]$} & $\lambda=0.9995$ \\
\hline I & [11] & $\begin{array}{l}\text { A cascade of } 2 \text { nd order notch filters was } \\
\text { used instead of a higher order notch } \\
\text { filter for stability, } \rho=0.96, \rho_{0}=0.7 \\
p=2, \rho \text { was reduced in steps of } 0.005 \\
\text { per sample }\end{array}$ \\
\hline $\mathrm{J}$ & {$[12]$} & $\begin{array}{l}\mu=0.01, K_{2} \text { was reduced from } 0.5 \text { to } \\
0.05 \text { in steps of } 0.0002 \text { per sample }\end{array}$ \\
\hline K & {$[5]$} & $\alpha=0.96, \lambda=0.995$ \\
\hline $\mathbf{L}$ & {$[13]$} & $\begin{array}{l}\text { Double precision arithmetic was used } \\
\text { for stability, } \lambda=1.0, \rho=0.96\end{array}$ \\
\hline M & {$[1]$} & $\begin{array}{l}\text { The preset threshold in FFT was } \\
\text { chosen to give minimum MSE }\end{array}$ \\
\hline $\mathrm{N}$ & {$[4]$} & $\alpha=0.995$ \\
\hline \multicolumn{3}{|c|}{$\begin{array}{c}K_{0}=-\cos (\omega), \omega \text { is the DSI frequency to be rejected } \\
\text { by a single stage }\end{array}$} \\
\hline
\end{tabular}

\section{RESULTS AND DISCUSSION}

THE input signal generated by the method outlined 1 earlier was applied to the various filters described in Section 2 (Figure 3) and their performance was investigated. In addition, those suggested by Feser et al. [1] and Hartje [4] for rejection of DSI in on-site PD measurements (designated as methods $\mathrm{M}$ and $\mathrm{N}$ ) have also been evaluated. Of these, method $\mathrm{K}$ [5] uses an adaptive method for updating the coefficients of the cascaded 2nd order 
lattice IIR notch filter. This filter is faster and has better discriminating power when compared with other notch filters. Hence, it is of interest to see how this filter performs when adaptation is not employed. However, for the implementation of this idea, the DSI must be determined by a separate procedure. This approach is designated as method $\mathrm{A}$. The procedures and parameters used for simulation of all these filters are listed in Table 2.

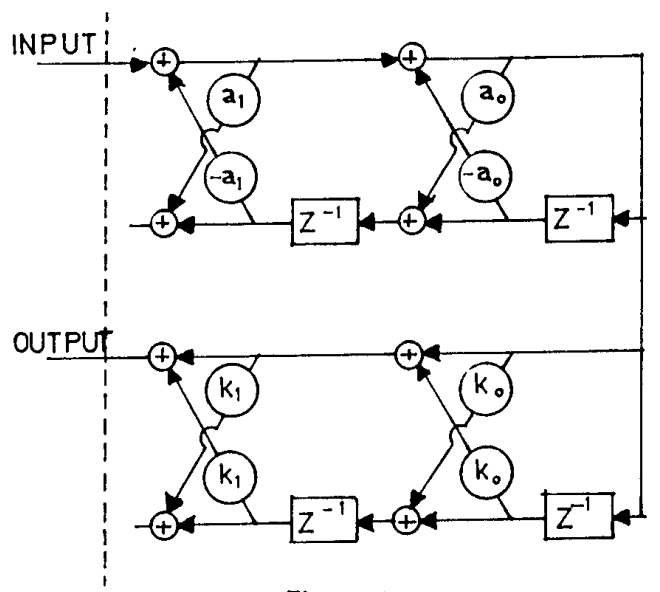

Figure 5.

Structure of one-stage 2 nd order lattice IIR notch filter. $k_{1}=1, a_{1}=\alpha, a_{0}=k_{0}$.

\subsection{CHOICE OF FILTER ORDER}

For realizing a digital filter, it is necessary to choose the filter order. This depends on the number of DSI present in the input signal and the filter structure. It should be noted that, as the filter order increases, more DSI can be rejected. The variation of MSE of the various nonadaptive and adaptive methods with respect to filter order are shown in Figure 6(a) and (b). It can be seen from Figure 6(a) that the MSE of method A exhibits a pronounced minimum in the order of 16 . This is also the ideal value of the filter order required to track the 8 DSI present in the simulated signal (with each 2nd order section in the cascade filtering a single DSI independently). That order which results in minimum MSE is the optimal filter order. A filter with order more than the optimum not only eliminates the DSI present completely but also tries to track noise present (white) assuming it to be DSI. This results in an increase in MSE. In the case of transversal and lattice filters (methods B and C), which are based on linear prediction [14], the MSE decreases monotonically with the filter order. As these filters are sensitive to noise, they require larger filter orders to track low INR signals. The linear-phase filter (method D), which is also based on linear prediction, follows the same trend but gives slightly better results.
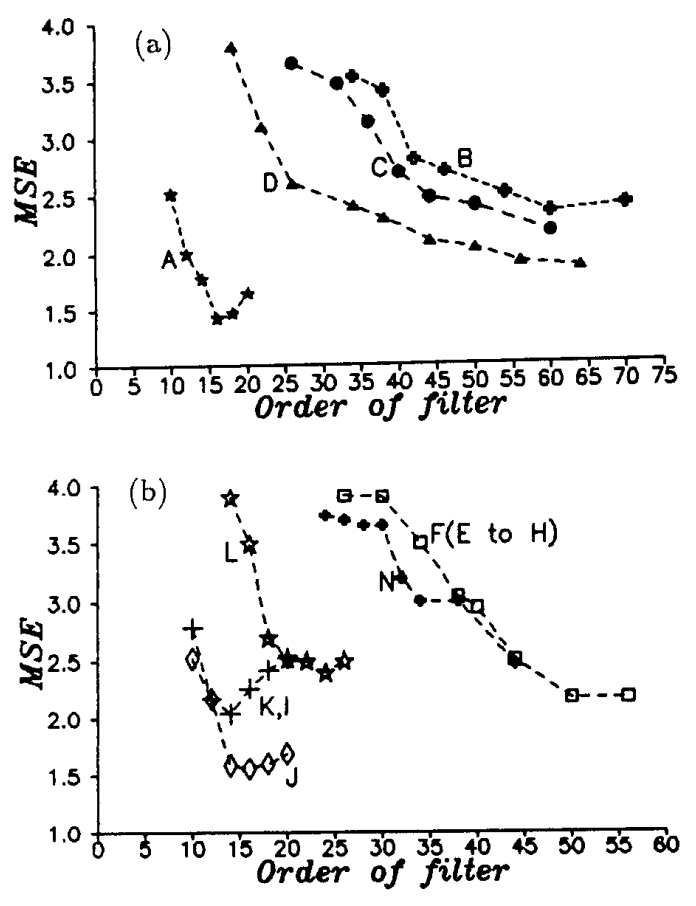

Figure 6.

Variation of MSE with filter order. (a) Nonadaptive methods, (b) Adaptive methods.

In adaptive methods (Figure 6(b)) also, the cascaded IIR notch filters (methods I, J and K) exhibit pronounced MSE minima when compared with the canonical IIR notch filter (method L). As the linear prediction methods $(\mathrm{E}, \mathrm{F}, \mathrm{G}, \mathrm{H}$ ) had similar behavior, they have been represented by the one having the best performance (method F). It can be seen from Figure 6(a) and (b) that the performances of both adaptive and nonadaptive linear prediction methods is very similar.

Although methods $\mathrm{A}$ and $\mathrm{K}$ use the same lattice IIR notch filter structure, the minimum in the latter occurs at 14 instead of 16 because the adaptive algorithm could not track a DSI with low INR. This behavior is also observed in method I. Method J (Kwan and Martin [12]) gives the best performance among adaptive filters; however, it was found to have a marked sensitivity to the adaptation parameters chosen. The parameters suggested by them could not be used because of instability and hence were modified as shown in Table 2. The value of the required adaptation parameters to be used depend strongly on the input signal and can only be chosen by trial and error. Hence, this method does not have the robustness required for practical applications.

Because of the prominent minima exhibited by the cascaded IIR notch filters (methods A, I, J and K), the op- 
timum filter order easily can be chosen as equal to twice the number of DSI. In lattice and transversal filters, it emerges that the filter order has to be a tradeoff between performance, and time taken to process the data which increases with the filter order.
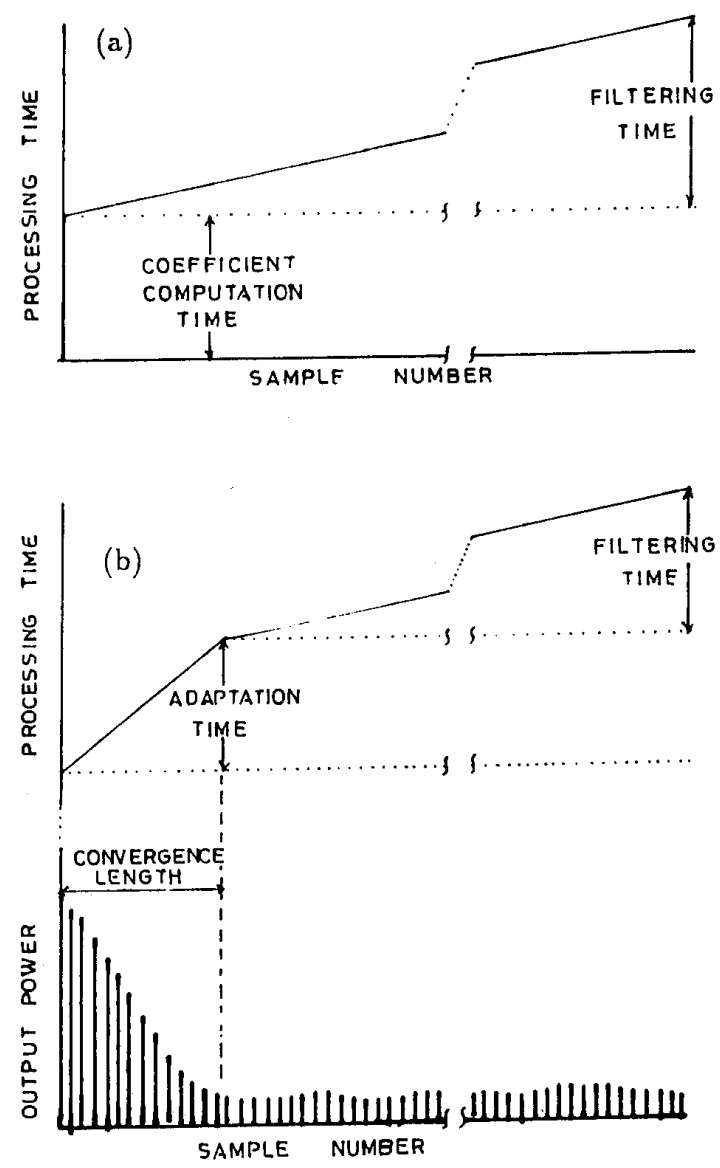

Figure 7.

Illustration of different times involved in processing. (a) Nonadaptive. (b) Adaptive.

\subsection{COMPARISON OF PROCESSING TIMES}

Another equally important criterion is the processing time. The input data record extending over a few power frequency cycles is usually $>128 \mathrm{k}$ samples due to the high sampling rate. Hence, a fast processing filter is desired. For clarity, it is convenient to introduce the following terms with reference to Figure $7(\mathrm{a})$ and (b) for nonadaptive and adaptive methods respectively. In nonadaptive filters, the total processing time equals the initial coefficient computation time plus the filtering time.
For convenience, the filtering time expressed as that for 1000 samples will be used.

In adaptive filters, Figure $7(\mathrm{~b})$, the adaptation is stopped after the output power reached a steady minimum value, thereby indicating convergence. If the adaptation is continued further, it only results in more processing tire without any improvement in performance. The number of samples required to reach this condition is named the convergence length. It is difficult to determine exactly the convergence length, because the steady output power to which it converges, is not known a priori. Hence, only approximate values will be given.

In adaptive filters, the total processing time equals the adaptation time plus the filtering time. Here again, their rate expressed for 1000 samples are used. It can be seen from Figures 7(a) and (b) that the filtering time increases linearly with the record length and becomes dominant for large input records as is the case in PD measurements. Hence, only this quantity need be considered.

The filtering time depends on the digital filter structure, i.e., the number of multiplications, additions and register transactions performed on each sample. It is difficult to break this filtering time into its components because each of the above operations is performed differently according to the speed and architecture of the processor used. Here, the pertinent times taken by an IBM PC AT 386 (16 MHz and with Math coprocessor) using compiled ' $\mathrm{C}$ ' language program for file input, output and filtering, are given. The time taken to perform a file read, FFT, IFFT and file write on a block of 1024 samples on the same computer took $1.7 \mathrm{~s}$. This Figure can be used for estimating the filtering time on other platforms.

Figures 8(a) and (b), show the variation of MSE with filtering time for nonadaptive and adaptive methods. It can be seen that the lattice and transversal filters, which need long filter orders, obviously take longer. Method E showed only a broad minimum with high MSE while method L takes a long time for acceptable MSE; hence, they are not shown in Figure 8(b). The cascade IIR notch filters not only have better performance but also are fast. Of these, method A using IIR lattice notch filter, gives the best results.

The summary of all the nonadaptive and adaptive methods for best/acceptable performance is given in Tables 3 and 4. It can be seen that the MSE in methods B, C, D (Table 3) and E, F, G, H, L (Table 4) are high and the 

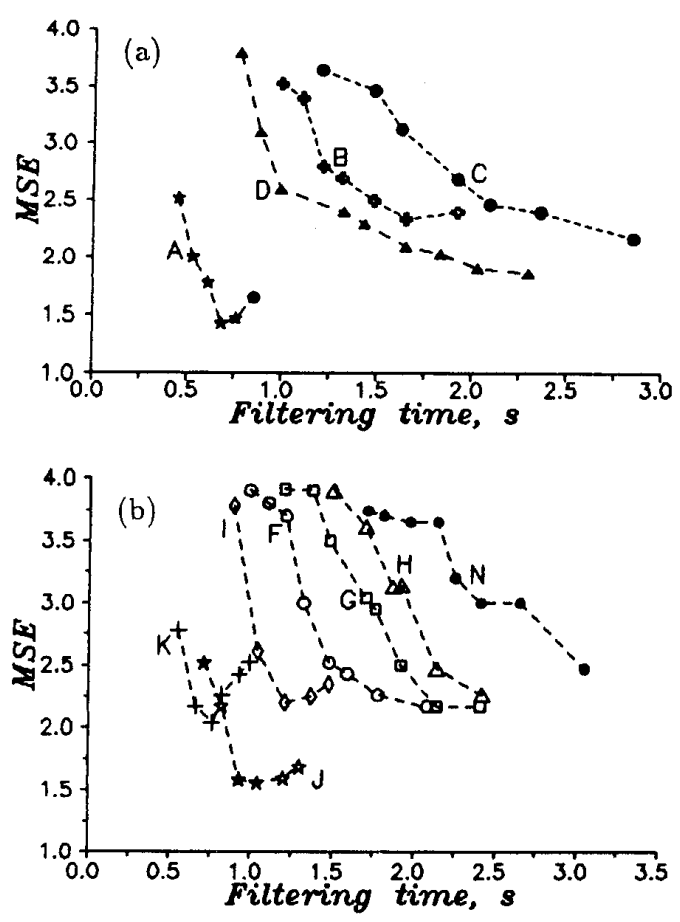

Figure 8.

Variation of MSE with filtering time. (a) Nonadaptive. (b) Adaptive.

Table 3.

Best acceptable performance of nonadaptive methods

\begin{tabular}{|c|c|c|c|c|}
\hline \hline Method & $\begin{array}{c}\text { Filter } \\
\text { order }\end{array}$ & $\begin{array}{c}\text { Time to } \\
\text { compute } \\
\text { filter } \\
\text { coeff (s) }\end{array}$ & $\begin{array}{c}\text { Filtering } \\
\text { time for } \\
1000 \text { samples } \\
\text { (s) }\end{array}$ & MSE \\
\hline A & 16 & 6.0 & 0.75 & 1.43 \\
B & 60 & 7.2 & 1.65 & 2.34 \\
C & 60 & 7.2 & 2.85 & 2.17 \\
D & 60 & 15.0 & 2.3 & 1.87 \\
\hline \hline
\end{tabular}

optimum filter order cannot be chosen easily even when the number of DSI present is known. Hence, it can be concluded that these are not suitable for practical applications and only the cascaded notch filters (methods A, $\mathrm{I}$ and $\mathrm{K}$ ) emerge as the most suitable.

\subsection{EFFECT OF IMPULSIVE DISTURBANCES}

In on-site measurements, it is difficult to avoid very large transient pulses; a number of those may occur in
Table 4.

Best acceptable performance of adaptive methods

\begin{tabular}{|c|c|c|c|c|c|}
\hline \hline & Filter & $\begin{array}{c}\text { Adaptation } \\
\text { order } \\
\text { 1k samp for } \\
\text { (s) }\end{array}$ & $\begin{array}{c}\text { Approximate } \\
\text { number of } \\
\text { samples for } \\
\text { convergence }\end{array}$ & $\begin{array}{c}\text { Filtering } \\
\text { time for } \\
\text { 1k samples } \\
(\mathrm{s})\end{array}$ & MSE \\
\hline $\mathrm{E}$ & 50 & 2.74 & 3000 & 1.35 & 3.17 \\
$\mathrm{~F}$ & 60 & 7.35 & 2000 & 2.1 & 2.2 \\
$\mathrm{G}$ & 50 & 6.2 & 3000 & 2.14 & 2.17 \\
$\mathrm{H}$ & 50 & 11.2 & 2000 & 2.4 & 2.2 \\
$\mathrm{I}$ & 14 & 3.5 & 4000 & 1.37 & 2.2 \\
$\mathrm{~J}$ & 16 & 7.85 & 5000 & 1.05 & 1.55 \\
$\mathrm{~K}$ & 14 & 1.7 & 7000 & 0.76 & 2.04 \\
$\mathrm{~L}$ & 22 & 4.9 & 4000 & 3.0 & 2.5 \\
$\mathrm{M}$ & - & 4.5 & - & 4.5 & 1.4 \\
$\mathrm{~N}$ & 30 & 4.5 & 3000 & 2.14 & 3.6 \\
\hline \hline
\end{tabular}

each cycle due to persistent arcing, switching, power electronics, etc. In order to test the sensitivity of the filter to such additional inputs, impulses with amplitude covering the full range were superimposed on the input record every $0.5 \mathrm{~ms}$ to represent a severe condition. A study of the pertinent output records revealed that the nonadaptive method A was stable and tracked all the DSI. However, the adaptive methods $I$ and $K$ were found to be sensitive and did not track a few of the DSI when these pulses occurred during adaptation of the filters. Hence, these adaptive methods can only be used when the initial part (convergence length) of the record is free from such large pulses. The application of these adaptive methods will also be limited with much DSI and low INR, as already mentioned.

\section{CRITICAL EVALUATION OF METHOD A}

\subsection{COMPARISONS WITH METHODS $N$ AND $M$}

TN method A, the detection and resolution of DSI depends on the length of FFT used for spectral analysis. A length of 4096 samples was found to be adequate in detecting DSI even with very low INR. Hence, when compared with other methods, method A has many advantages:

1. good stability against impulsive disturbances,

2. simple and fast algorithm to compute the filter coefficients,

3. optimal choice of filter order for rejection of all the DSI present (even with low INR) resulting in minimum MSE and 
4. small filtering time.

It is interesting to compare the performance of method A with the methods proposed earlier by Feser et al. [1] and Hartje [4] ( $M$ and N) for on-site PD measurements. In method $M$, the input data record is split into smaller blocks of 1024 samples. The FFT of each block is taken after multiplying it by a window function to give better spectral resolution. The amplitudes of the DSI, which exceed a preset threshold, are set to zero. Next, an IFFT is taken on each of these blocks to get back the PD pulses.

In the method $N$, an adaptive backward prediction lattice filter used in speech processing, has been applied to reject the DSI. Such adaptive filters, based on linear prediction, have fast convergence and hence are used widely in tracking nonstationary signals like speech. However, they are sensitive to noise and cannot track low INR DSI. Higher orders had to be used in these comparisons, due to the presence of many DSI. These results are shown in Figures $6(\mathrm{~b})$ and $8(\mathrm{~b})$. The behavior of this filter is similar to other linear prediction methods. Although its MSE is slightly less when compared with other linear prediction methods for the same order, the filtering time is longer.

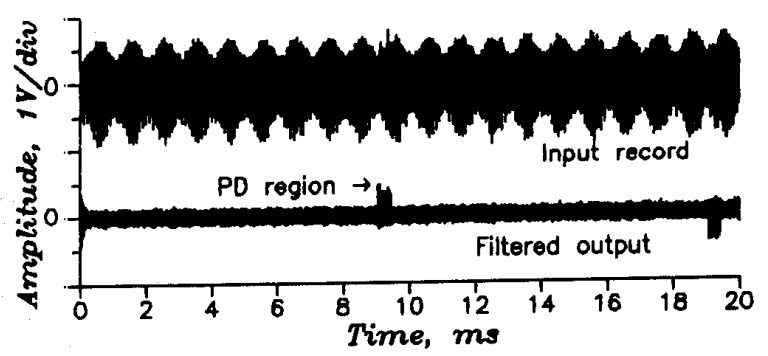

Figure 9.

Filtering of synthesized data by method A.

The pulse resolution capabilities of methods $A, N$ and $\mathrm{M}$ are also of interest. Only a two-pulse situation (original PD and its reflection) in a cable system has been reported by Borsi [15]. Figure 9 shows the input signal as described in Section 3 and the filtered output using method $A$. The burst of PD pulses which is not visible in the input, can easily be seen after filtering. An expanded view of the $P D$ region is given in Figure 10 for methods $A, N$ and $M$. As mentioned in Section 3, a good filter should reproduce each of the original PD pulses with least distortion. The distortion in method $\mathrm{N}$ is higher when compared to methods $\mathrm{A}$ and $\mathrm{M}$ as the oscillations which occur after each pulse are large enough to distort the next pulse. This prevents many PD pulses from being recognized, thereby

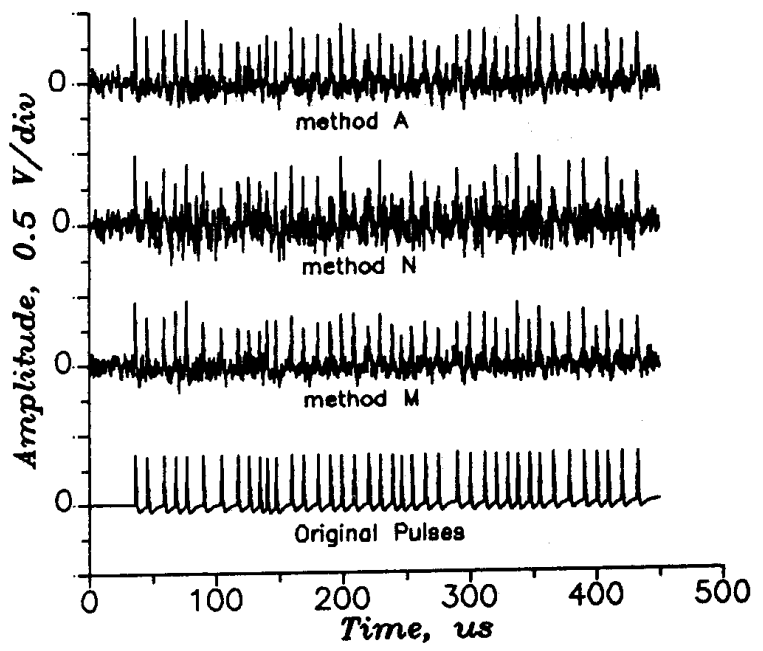

Figure 10.

Expanded view of PD region shown in Figure 9.

losing important diagnostic information. Borsi [15] has discussed the PD pulse resolving capability of this filter with and without continuous adaptation. Borsi concludes that the resolution is better without continuous adaptation i.e., the adaptation is stopped well before the arrival of PD pulses. However, in the results presented (Figures 6, 7 and 12 in [15]) there is considerable distortion in the pulse shape as well as oscillations after the pulse, even when adaptation is stopped. It was found that, on increasing the filter order, the oscillations decreased. But this results in longer processing time. Another disadvantage of all adaptive methods including method $\mathrm{N}$ is that the exact time at which adaptation has to be stopped cannot be estimated $i$ ?? a priori and requires operator intervention.

Table 5. Performance of methods $A$ and $M$

\begin{tabular}{|c|c|c|c|c|}
\hline \hline \multirow{2}{*}{$\begin{array}{c}\text { Number } \\
\text { of DSI }\end{array}$} & \multicolumn{2}{|c|}{ MSE } & \multicolumn{2}{c|}{$\begin{array}{c}\text { Filtering time for } \\
1000 \text { samples (s) }\end{array}$} \\
\hline 5 & 1.2 & 1.2 & 0.47 & 4.65 \\
10 & 1.6 & 1.56 & 0.85 & 4.65 \\
15 & 1.88 & 1.84 & 1.25 & 4.65 \\
20 & 2.28 & 2.08 & 1.65 & 4.66 \\
25 & 2.72 & 2.16 & 2.0 & 4.66 \\
\hline
\end{tabular}

From Figure 10, it can be seen that both methods A and $M$ reproduce well the original pulses. This is reflected also in their MSE given in Tables 3 and 4. From observation, it found that MSE has to be $<2.0$ for acceptable pulse distortion. The filtering time taken in method $\mathrm{A}$ is 
small when compared to method M. As the number of DSI increases, the filtering time in the method $\mathrm{A}$ increases linearly, while that in $\mathrm{M}$ almost stays constant. In order to further study this behavior, another input signal was generated using the same method outlined earlier. However, for simplicity, the DSI were spaced equally throughout the frequency range of $1 \mathrm{MHz}$ and had equal power (INR $=3 \mathrm{~dB}$ ) but random phases. The results are shown in Table 5, for different number of DSI. As the number of DSI increase, the distortion in both methods and the time taken in method A increases. Considering that, in on-site PD measurements, the number of DSI usually is $\leqslant 15$, method A scores over method M. It should be remarked that in a later paper, König et al. [16] have proposed to use adaptive transversal filters instead of the continuous FFT method $(M)$ in order to reduce the processing time.

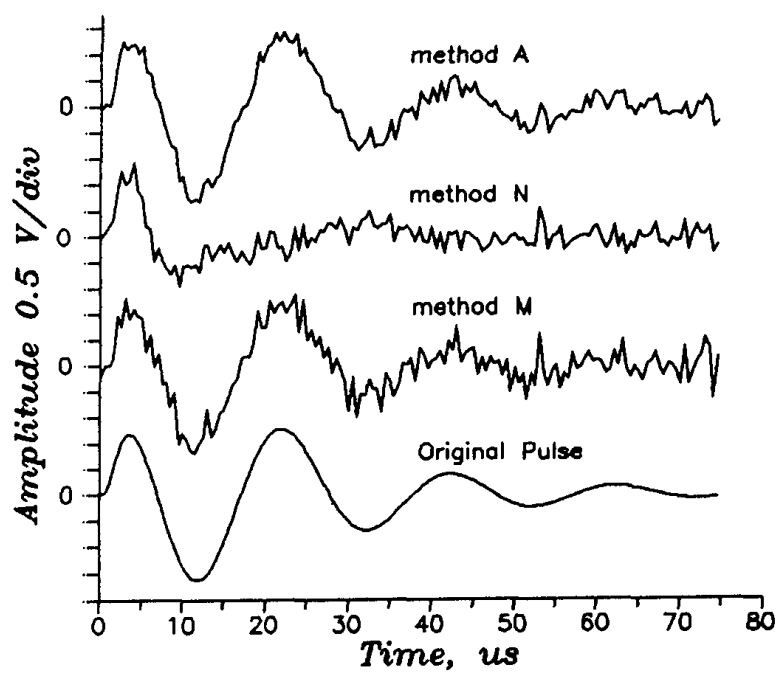

Figure 11.

Enlarged view of an oscillatory $\mathrm{PD}$ pulse and its filtered outputs.

\subsection{EFFECT OF OSCILLATORY PD PULSES}

So far, all the simulation and comparisons relate to fastrising narrow $\mathrm{PD}$ pulses having a time constant of $50 \mathrm{~ns}$. The behavior of the filtering methods for other types of PD pulses with different widths and oscillations are also of interest. This was studied by superposing pulses of different widths in the synthesized input signal. The pulses were generated by passing both exponential and damped oscillatory pulses of different time constants through coupling circuit, simulated by a 2nd order Butterworth bandpass filter $(30$ to $300 \mathrm{kHz}$ ). Figure 11 shows a typical oscillatory pulse $(50 \mathrm{kHz}$, time constant $20 \mu \mathrm{s})$ and its filtered output by methods $\mathrm{A}, \mathrm{M}$ and $\mathrm{N}$. It was seen that oscillatory pulses and pulses of larger widths, cannot be reproduced by linear prediction methods, while methods $A$ and $M$ reproduce pulses of all widths with little error.

Hence methods $\mathrm{A}$ and $\mathrm{M}$ can be used for all types of insulation systems which may produce PD pulses of different shapes.

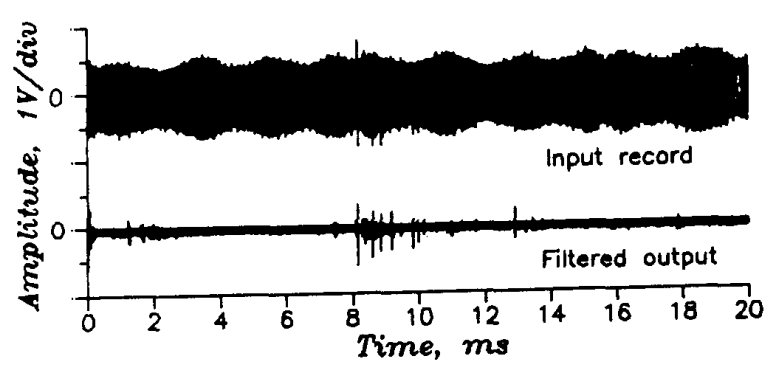

Figure 12.

Real DSI and filtered output using method A (sampling time $500 \mathrm{~ns}$ ).

\subsection{PERFORMANCE WITH REAL DSI}

The performance of method A was tested with real DSI. These signals were recorded outdoor using a wide-band antenna $(10$ to $300 \mathrm{kHz}$ ), at a sampling rate of $500 \mathrm{~ns}$ (12 bit resolution). An FFT of this signal showed the presence of 4 DSI with frequency $24.2,72.6,612$ and 675 $\mathrm{kHz}$, of which the third was a powerful local radio station ( $20 \mathrm{~dB}$ above the rest). Figure 12 shows one such record and its filtered output using method $\mathrm{A}$. It can be seen that on filtering the DSI, many large random impulsive interferences become visible.

In addition to the above the effectiveness of method $A$ was tested with DSI of large magnitudes. As the amplitude of the DSI increases, its base in the frequency spectrum relatively increases and therefore requires a wider notch for optimal rejection. The increase in the notch width was obtained by decreasing $\alpha$ from 0.98 to 0.95 . With this notch width, the filter was able to reject the DSI (10 $\times$ the height of the PD pulse) sufficiently well so that the $\mathrm{PD}$ pulses were visible. Also, the filter was tested with an input synthesized by using real modulating signals instead of the constant $1 \mathrm{kHz}$ modulating frequency. These modulating signals were recorded from an audio amplifier output. The results were similar to that described in Section 4. 


\subsection{EFFECT OF INCREASE IN SAMPLING RATE}

In many applications, it is necessary to study the PD pulse shapes in greater detail, for which the sampling period has to be reduced to $\leqslant 100 \mathrm{~ns}$. As the sampling rate increases, the Nyquist frequency increases. Hence, for a given length of data, the frequency resolution decreases and width of the DSI in the frequency spectrum decreases relatively. If a filter has to effectively reject a DSI, then the notch width must be reduced to the required bandwidth of the DSI. The signal distortion increases if the notch width is greater than the optimum, as a portion of the wide-band signal (PD) is also lost.

The reduction of notch width can easily be achieved in method A (Section 2) by increasing $\alpha$. For example, if the sampling time is reduced from 500 to $100 \mathrm{~ns}$, a change in $\alpha$ from 0.98 to 0.995 was found to yield optimum results, without any change in the filter length. Such a simple mechanism of decreasing the bandwidth of the notch is not present in linear prediction methods. A major part of the wide-band signal is lost due to rejection of wide bandwidths resulting in reduction of amplitude and distortion of all the pulses.

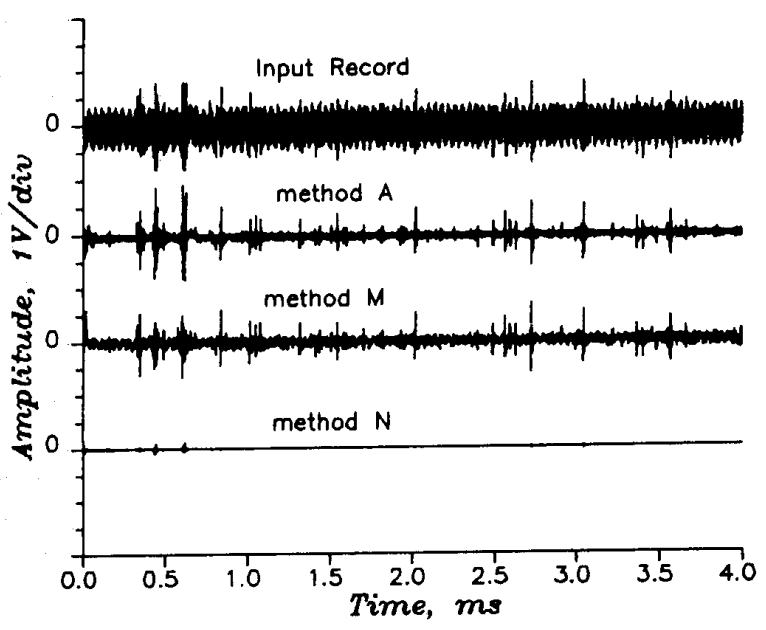

Figure 13.

Filtered outputs due to real DSI (Sampling time $100 \mathrm{~ns}$ ).

A plot of real DSI recorded outdoors using a wide-band $(10$ to $300 \mathrm{kHz}$ ) antenna at $100 \mathrm{~ns}$ is shown in Figure 13 with the corresponding outputs of methods $A, M$ and $N$. The output pulses in method $\mathrm{N}$ has drastic reduction in amplitudes due to reasons mentioned earlier and also to the relative increase in the pulse width at higher sampling rates.

\subsection{PROSPECTS FOR HARDWARE IMPLEMENTATION}

In the simulations done so far, floating-point arithmetic was used during calculations. This arithmetic involves long calculation times in comparison with fixedpoint arithmetic ( 16 bits). Although the latter requires careful attention to round-off errors and register overflows, calculations are simple and require less hardware. In this regard, method A was simulated using fixed-point arithmetic (16 bits) and no instabilities were found, with the input signal described in Section 3. However, the MSE increased from 1.43 to 1.78 due to roundoff errors and necessity to reduce the pole radii ( $\alpha=0.96$, Figure 5) to prevent instabilities. Hence, a hardware implementation is possible with 16-bit fixed-point arithmetic processors (with an ALU of 32 bits), which are faster and cheaper than floating-point processors.

\section{CONCLUSIONS}

$T_{\mathrm{HE}}$ analysis and results presented in this paper leads 1 to the following conclusions:

From a systematic study of different adaptive and nonadaptive digital filters, it was possible to identify the most suitable types for rejecting the DSI present in PD measurements. For evaluating these filters, the PD pulse distortion introduced by the filter while rejecting the DSI and the filtering time have been chosen as criteria. The distortion was characterized by the mean square error (MSE) between the input and output PD pulses as it was sensitive to the PD pulse distortion and the residual DSI.

The nonadaptive linear-prediction methods ( $\mathrm{B}, \mathrm{C}$ and D) do not give satisfactory results (high MSE) when DSI with low INR are present. Also, the choice of filter order in these methods is based on a tradeoff between the MSE and the processing time. The adaptive linear prediction methods (E, F, G and H) also suffer from the same disadvantages.

Method N, which is also based on linear prediction has similar performance. The PD pulse distortion is considerable and long filter orders were required to reduce MSE to acceptable limits, which increases the processing time.

Methods A, I, J and K using a cascaded 2nd order IIR notch filter have prominent MSE minima when compared 
with the canonical IIR notch filter (method L). However, the adaptive cascade IIR notch filters (methods I, J and K) were sensitive to impulsive disturbances and also could not track low INR DSI, when compared with method A.

Methods $A$ and $M$ have good DSI rejection and the least $\mathrm{PD}$ pulse distortion. But the processing time and MSE in the former is less when the number of DSI are not more than 15 .

In addition, method $\mathrm{A}$ has a simple procedure to compute the filter order and its coefficients without operator intervention, a low MSE, good stability against impulsive disturbances and small processing time. This method can be used in applications requiring high sampling rates which are necessary for good PD resolution and also for diagnostics in various types of insulation systems. For speeding up further, it is also possible to use fixed-point processors for hardware implementation of this filter.

Hence, the proposed method $\mathrm{A}$ emerges as the best for on-site PD measurements.

\section{ACKNOWLEDGMENT}

$\mathrm{T}$

HE first author wishes to thank Mr. L. Satish for his help during the preparation of this manuscript. The authors also thank Mr. R. S. S. Aradhya of CPRI Bangalore, for his help in recording real DSI data.

\section{REFERENCES}

[1] K. Feser, G. König, J. Ott, and P. Seitz, "An Adaptive Filter Algorithm for On-site Partial Discharge Measurements", Conference Record of the 1988 International Symposium on Electrical Insulation, Boston, Ma, June 5-8, 1988.

[2] H. Borsi, M. Hartje, "New Methods to Reduce the Disturbance Influences on the In Situ -Partial Discharge (Pd)- Measurement and Monitoring", Paper $15.10,6$ th International Sym. on High Voltage Eng., New Orleans, Aug. 28 - Sep 1, 1989.

[3] A. V. Oppenheim and R. W. Schafer, Digital Signal Processing, Prentice-Hall International, Inc., 1989.

[4] M. Hartje, Erfassung von Teilentladungen an Leistung Stransformatoren im Netzbetrieb, Dissertation Universtität Hannover, 1989.
[5] N. I. Cho, C. H. Choi and S. U. Lee, "Adaptive Line Enhancement by Using an IIR Lattice Notch Filter", IEEE Transactions on Acoustic, Speech and Signal Processing, Vol. 37, No. 4, pp. 585-589, Apr. 1989.

[6] I. Novak, "Statistical Analysis of High-accuracy Measurement of Radio-frequency Carrier Signals from DFT Spectra", Conference on Precision Electromagnetic Measurements, Ottawa, Canada, June 11-14, 1990.

[7] S. J. Orfanidis, Optimum Signal Frocessing: An Introduction, 2nd Edition, McGraw-Hill Book Company, 1990 .

[8] S. L. Marple, "Fast Algorithms for Linear Prediction and System Identification Filters with Linear Phase", IEEE Transactions on Acoustic, Speech and Signal Processing, Vol. 30, No. 6, Dec. 1982.

[9] G. Carayannis, D. G. Manolakis and N. Kalouptsidis, "A Fast Sequential Algorithm for Leastsquares Filtering and Prediction", IEEE Transactions on Acoustic, Speech and Signal Processing, Vol. 31, No. 6, Dec. 1983.

[10] B. Friedlander, "Lattice Filters for Adaptive Processing", Proceedings of the IEEE, Vol. 70, No. 8, Aug. 1982.

[11] A. Nehroi, "A Minimal Parameter Adaptive Notch Filter With Constrained Poles and Zeros", IEEE Transactions on Acoustic, Speech and Signal Processing, Vol. 33, No. 4, Aug. 1985.

[12] T. Kwan and K. Martin, "Adaptive Detection and Enhancement of Multiple Sinusoids Using a Cascade IIR Filter", IEEE Transactions on Circuits and Systems, Vol. 36, No. 7, July 1989.

[13] J. M. Travassos-Romano, and M. Bellanger, "Fast Least Squares Adaptive Notch Filtering", IEEE Transactions on Acoustic, Speech and Signal Processing, Vol. 36, No. 9, Sep. 1988.

[14] J. Makhoul, "Linear Prediction: A Tutorial Review", Proceedings of the IEEE, Vol. 63, No. 4, Apr. 1975.

[15] H. Borsi, "Digital Location of Partial Discharges (Pd) in HV Cables", Document 33-91 (WG 03) 23 IWD, CIGRE WG 33.03 Meeting, June 4-5, 1991, St. Petersburg. Published in IEEE Trans. on Electrical Insulation, Vol. 27, No. 1, pp. 28-36, Feb 1992. 
[16] G. König, U. Kopf, and K. Feser, "Methods to Improve On-site Partial Discharge Measuring Instruments", 7th International Sym. on High Voltage Eng., Paper 73.02, Dresden, Germany, Aug 26-30 1991.

[17] W. S. Zaengl, P. Osvath, and H. J. Weber, "Correlation between the Bandwidth of PD-detectors and its Inherent Integration Errors", Revue Generale Electricité, No 4, April 1989.
[18] J. P. Steiner and W. L. Weeks, "A New Method for Locating Partial Discharges in Transformers", Conference on Electrical Insulation and Dielectric Phenomena, pp. 275-281, Oct. 1990.

Manuscript was received on 20 February 1992, in final form 8 September 1992. 